\title{
Valvotomia Mitral Percutânea: Quarta Dilatação
}

\author{
Roberto Ramos Barbosa', Vinícius B. C. Esteves', Nísia Lyra Gomes', Sérgio Luiz N. Braga', \\ Auristela I. O. Ramos ${ }^{1}$, Mercedes Maldonado', Sergio Tavares ${ }^{1}$, Cleverson N. Zukowski', \\ Zilda Machado Meneghelo', César A. Esteves ${ }^{1}$
}

\section{RESUMO}

Paciente do sexo feminino, 37 anos, portadora de estenose mitral de etiologia reumática, submetida, com sucesso, a valvotomia mitral percutânea em 1996, 2000 e 2005. Após 41 meses do terceiro procedimento, além de episódios de fibrilação atrial paroxística, voltou a apresentar dispneia aos moderados esforços. O estudo ecocardiográfico demonstrou gradiente transvalvar médio de $8 \mathrm{mmHg}$, pressão sistólica da artéria pulmonar de $55 \mathrm{mmHg}$, área valvar mitral de $1 \mathrm{~cm}^{2}$ e escore de Wilkins de 10 pontos. Em decorrência da evidência de reestenose clínica e ecocardiográfica, indicou-se novo procedimento percutâneo, realizado sem intercorrências, porém obtendo-se resultado subótimo.

DESCRITORES: Estenose da valva mitral. Dilatação com balão. Resultado de tratamento.
D esde 1984, quando foi inicialmente descrita por Inoue et al. ${ }^{1}$, a valvotomia mitral percutânea (VMP) tem tido suas indicações ampliadas, tornando-se o método terapêutico de escolha para pacientes selecionados, portadores de estenose mitral grave. ${ }^{2}$

Uma VMP prévia (assim como uma comissurotomia cirúrgica prévia) não constitui por si só contraindicação para nova VMP, sendo a avaliação anatômica, obtida pelo escore ecocardiográfico de Wilkins ${ }^{3}$, e a condição clínica do paciente os principais parâmetros para a indicação do procedimento.

Estudos referentes aos resultados de segundas e terceiras dilatações são escassos na literatura ${ }^{4}$, e não encontramos relatos de pacientes que tenham sido submetidos a mais de três procedimentos.

\footnotetext{
1 Instituto Dante Pazzanese de Cardiologia - São Paulo, SP, Brasil. Correspondência: Roberto Ramos Barbosa. Avenida Dante Pazzanese, 500 - Vila Mariana - São Paulo, SP, Brasil - CEP 04012-909 E-mail: roberto.rb@cardiol.br

Recebido em: 7/3/2011 • Aceito em: 10/5/2011
}

\section{ABSTRACT}

\section{Percutaneous Mitral Valvotomy: Fourth Dilatation}

Thirty-seven-year-old female, previously diagnosed with mitral valve stenosis due to rheumatic fever, who had successfully undergone percutaneous mitral valvotomy in 1996, 2000 and 2005. Forty-one months after the third procedure, in addition to episodes of paroxystic atrial fibrillation, the patient had dyspnea after ordinary exertion. Echocardiographic evaluation revealed a mean transvalvular gradient of $8 \mathrm{mmHg}$, pulmonary artery systolic pressure of $55 \mathrm{mmHg}$, a mitral valve area of $1 \mathrm{~cm}^{2}$, and a Wilkins score of 10 points. With the evidence of clinical and echocardiographic mitral restenosis, a new percutaneous procedure was performed, with no periprocedural complications, however, achieving suboptimal outcome.

KEY-WORDS: Mitral valve stenosis. Balloon dilatation. Treatment outcome.

Relatamos o caso de uma paciente submetida eletivamente a VMP pela técnica de Inoue pela quarta vez.

\section{RELATO DO CASO}

A paciente recebeu o diagnóstico de estenose da valva mitral em 1996, aos 22 anos de idade, e foi encaminhada para acompanhamento no ambulatório especializado em valvopatias do Instituto Dante Pazzanese de Cardiologia, em São Paulo (SP, Brasil). Apresentava dispneia aos esforços (classe funcional II-III), e o exame físico demonstrava sopro diastólico em ruflar 3+/4+ em ápex e frêmito diastólico em foco mitral. Eletrocardiograma mostrava ritmo sinusal, com sobrecarga atrial esquerda. Ecocardiograma demonstrava área valvar mitral de $0,73 \mathrm{~cm}^{2}(\mathrm{PHT})$, fração de ejeção do ventrículo esquerdo de $77 \%$ (Simpson), átrio esquerdo de $53 \mathrm{~mm}$, valva mitral com insuficiência discreta e gradiente diastólico máximo de 29 e médio de 17, escore de Wilkins de 9 pontos, e pressão sistólica de artéria pulmonar de $75 \mathrm{mmHg}$.

Confirmado o diagnóstico de estenose mitral grave, indicou-se a primeira VMP da paciente. O procedimento foi realizado com êxito em 29 de agosto de 1996. 
O ecocardiograma posterior à intervenção demonstrou área valvar mitral de 1,95 cm², gradiente diastólico pela valva mitral máximo de 14 e médio de 5, e pressão sistólica de artéria pulmonar de 50 mmHg (Tabela 1).

A paciente recebeu alta assintomática, com a prescrição de atenolol $25 \mathrm{mg} /$ dia e penicilina benzatina 1.200.000 UI intramuscular a cada 21 dias.

Após quatro anos assintomática, a paciente passou a apresentar dispneia aos esforços (classe funcional II), e relatou desejo de engravidar. Ecocardiograma demonstrava área valvar mitral de $0,9 \mathrm{~cm}^{2}$, fração de ejeção do ventrículo esquerdo de $63 \%$ (Simpson), átrio esquerdo de $54 \mathrm{~mm}$, valva mitral com insuficiência discreta e gradiente diastólico máximo de 18 e médio de 10, escore de Wilkins de 10 pontos, e pressão sistólica de artéria pulmonar de $45 \mathrm{mmHg}$. Caracterizada a reestenose valvar mitral clínica e ecocardiográfica, optou-se pela segunda VMP, que foi realizada com sucesso em 22 de setembro de 2000.

Em 2002, a paciente iniciou gestação, durante a qual permaneceu assintomática, com parto em 2003, sem intercorrências. Na ocasião, foi orientada a manter o uso de atenolol. Seu ecocardiograma demonstrava área valvar mitral de 1,6 $\mathrm{cm}^{2}$.

Em 2004, a paciente experimentou sua segunda gestação; nesse período, voltou a se queixar de dispneia aos esforços habituais (classe funcional II), e sua área valvar mitral ao ecocardiograma era de $1,34 \mathrm{~cm}^{2}$. Foi mantido o uso do atenolol, e tanto a gestação quanto o parto transcorreram sem quaisquer complicações materno-fetais.

Em 2005, a paciente apresentou piora clínica, evoluindo com dispneia em classe funcional II-III. Ao exame físico, o sopro diastólico mitral se acentuou; o ecocardiograma demonstrava área valvar mitral de $1 \mathrm{~cm}^{2}$, fração de ejeção do ventrículo esquerdo de $60 \%$
(Simpson), átrio esquerdo de $53 \mathrm{~mm}$, valva mitral com insuficiência discreta e gradiente diastólico máximo de 22 e médio de 13, escore de Wilkins de 10 pontos e pressão sistólica de artéria pulmonar de $58 \mathrm{mmHg}$. Mantinha ritmo sinusal ao eletrocardiograma.

Após recusa inicial, aceitou a indicação da terceira VMP, realizada em 5 de dezembro de 2005, novamente com resultados satisfatórios. A área valvar mitral passou para $1,8 \mathrm{~cm}^{2}$, e as pressões medidas $(\mathrm{mmHg})$ passaram de 50/18/32 para 42/18/20 em artéria pulmonar, e de 40 para 15 em átrio esquerdo. Novamente, recebeu alta hospitalar assintomática, sendo mantida a prescrição de atenolol e penicilina benzatina.

Um ano após a terceira dilatação, a paciente passou a apresentar episódios de fibrilação atrial paroxística, flagrados em gravação de Holter de 24 horas após queixas de palpitações eventuais. A área valvar mitral era de $1,67 \mathrm{~cm}^{2}$. Substituiu-se o atenolol por amiodarona 200 mg/dia, e foi iniciada anticoagulação oral com varfarina, que se mostrou eficaz no acompanhamento subsequente. Em 2009, a amiodarona foi substituída por propafenona.

A paciente retornou ainda no ano de 2009 com nova queixa de dispneia aos esforços (classe funcional II), e a área valvar mitral ao ecocardiograma era de 1,3 cm², com insuficiência mitral discreta. Com a suspeita de dissociação clínico-ecocardiográfica, foi indicado teste ergométrico, que evidenciou: ausência de alterações do segmento ST sugestivas de isquemia, capacidade aeróbica fraca (< $7 \mathrm{MET}$ ), e comportamento hemodinâmico anormal com queda da pressão arterial sistólica ao exercício.

Com o acompanhamento ambulatorial mais rigoroso após o teste ergométrico, em 2010 foi indicada a realização da quarta VMP, após confirmação diagnóstica de nova reestenose mitral clínica e ecocardiográfica. A dispneia evoluíra para classe funcional II-III, e o

TABELA 1

Alterações ecocardiográficas antes e após cada procedimento

\begin{tabular}{|c|c|c|c|c|}
\hline & $\begin{array}{c}\text { Primeira VMP } \\
1996\end{array}$ & $\begin{array}{l}\text { Segunda VMP } \\
2000\end{array}$ & $\begin{array}{l}\text { Terceira VMP } \\
2005\end{array}$ & $\begin{array}{c}\text { Quarta VMP } \\
2010\end{array}$ \\
\hline $\begin{array}{l}\text { Área valvar mitral } \\
\text { pré-pós }(\Delta), \mathrm{cm}^{2}\end{array}$ & $0,73-1,95(+1,22)$ & $0,9-1,7(+0,8)$ & $1-1,8(+0,8)$ & $1-1,4(+0,4)$ \\
\hline $\begin{array}{l}\text { Gradiente diastólico máximo } \\
\text { pré-pós }(\Delta), \mathrm{mmHg}\end{array}$ & $29-14(-15)$ & $18-17(-1)$ & $22-13(-9)$ & $15-12(-3)$ \\
\hline $\begin{array}{l}\text { Gradiente diastólico médio } \\
\text { pré-pós }(\Delta), \mathrm{mmHg}\end{array}$ & $17-5(-12)$ & $10-8(-2)$ & $13-7(-6)$ & $8-7(-1)$ \\
\hline PSAP pré-pós $(\Delta), \mathrm{mmHg}$ & $75-50(-25)$ & $45-40(-5)$ & $58-52(-6)$ & $55-60(+5)$ \\
\hline $\begin{array}{l}\text { Tamanho do átrio esquerdo } \\
\text { pré-pós }(\Delta), \mathrm{mm}\end{array}$ & $53-49(-4)$ & $54-45(-9)$ & $53-48(-5)$ & $51-49(-2)$ \\
\hline
\end{tabular}

PSAP = pressão sistólica de artéria pulmonar; VMP = valvoplastia mitral percutânea. 
ecocardiograma demonstrava área valvar mitral de $1 \mathrm{~cm}^{2}$, escore de Wilkins de 10 pontos e insuficiência mitral discreta, além de pressão sistólica de artéria pulmonar de $55 \mathrm{mmHg}$.

A quarta dilatação foi então realizada, aos 37 anos de idade, em 4 de maio de 2010, com balão de Inoue 27, sem intercorrências, porém obtendo resultados subótimos. Não foram encontradas dificuldades técnicas que pudessem ser atribuídas aos procedimentos anteriores. A área valvar mitral pós-procedimento passou para 1,4 $\mathrm{cm}^{2}(\mathrm{PHT})$, e a paciente recebeu alta hospitalar em classe funcional I.

No acompanhamento ambulatorial, a paciente voltou a apresentar piora da dispneia (classe funcional II) apenas tês meses após a realização da quarta VMP. Negava episódios de palpitações. A área valvar mitral pelo ecocardiograma era de 1,2 cm², com insuficiência mitral discreta. Orientou-se acompanhamento ambulatorial mais rigoroso. A medicação prescrita foi propranolol $40 \mathrm{mg}$ a cada 12 horas, furosemida $40 \mathrm{mg}$ uma vez por dia, varfarina (dose conforme a variação do INR, com o objetivo de mantê-lo entre 2 e 3) e penicilina benzatina 1.200.000 UI intramuscular a cada 21 dias.

A paciente persistiu com os sintomas, com má aderência à medicação prescrita, sendo proposta troca valvar mitral em novembro de 2010, aceita pela paciente em janeiro de 2011. Em 28 de fevereiro de 2011 recebeu prótese biológica mitral e foi realizada plastia da valva tricúspide, com sucesso, recebendo alta uma semana após, sem intercorrências.

\section{DISCUSSÃO}

A estenose valvar mitral como consequência de febre reumática ainda é um grande problema socioeconômico nos países em desenvolvimento. A VMP, procedimento inicialmente descrito há quase 30 anos, representa atualmente a opção terapêutica de escolha para grande parcela dos pacientes com estenose mitral grave isolada.

O sucesso da intervenção é definido por aumento da área valvar $\geq 25 \%$ da área inicial e área valvar mitral final $\geq 1,5 \mathrm{~cm}^{2}$, na ausência de insuficiência mitral grave, enquanto o insucesso é definido pela interrupção do procedimento causado por problema técnico ou pelo desenvolvimento de insuficiência mitral grave (3 ou 4+) após a insuflação do balão ou pela ocorrência de óbito. ${ }^{5}$

A reestenose pós-procedimento é uma realidade nos pacientes com estenose mitral submetidos a VMP, incidindo em torno de $18 \%$ e $22 \%$ em sete e dez anos, respectivamente. ${ }^{4,6}$ Considera-se reestenose ecocardiográfica uma área valvar mitral no seguimento tardio, avaliada pela planimetria e/ou pelo $\mathrm{PHT}<1,5 \mathrm{~cm}^{2}$, com perda $\geq 50 \%$ do ganho inicial. ${ }^{7}$ Reestenose clíni- ca é a definição dada para o retorno dos sintomas, avaliada pela classe funcional e comprovada pela avaliação ecocardiográfica.

São considerados fatores preditores de reestenose a área valvar mitral final pós-procedimento, o escore ecocardiográfico, a intensidade da calcificação valvar e/ou subvalvar, a idade e o ritmo de fibrilação atrial. ${ }^{8}$ Destaque especial quanto ao risco de reestenose foi dado, em recente análise de Cardoso et al. ${ }^{9}$, à área valvar mitral pré-procedimento e à presença de calcificação mitral acentuada. O sucesso parcial do procedimento, ou resultado subótimo (obtenção de área valvar $<1,5 \mathrm{~cm}^{2}$ na ausência de insuficiência mitral grave), também é um preditor de reestenose mitral precoce. ${ }^{10}$

Na população geral, o resultado da VMP a longo prazo é excelente, com sobrevida livre de eventos (inclusive reestenose) de 90\% (Tabela 2). Entretanto, em pacientes com escore de Wilkins $>8$ pontos, a sobrevida livre de eventos é menor $(68,9 \%$ em seguimento de quatro anos). ${ }^{10}$

Com relação a indicações repetidas de VMP em pacientes com reestenose clínica e ecocardiográfica, apesar da obtenção de áreas valvares mitrais finais menores, é possível atingir elevados índices de sucesso após uma segunda e até mesmo uma terceira intervenção, conforme estudo de Gomes et al. ${ }^{4}$. Entretanto, o pequeno número de pacientes submetidos a um terceiro procedimento não permitiu conclusões definitivas sobre essa indicação. Observa-se ainda redução significativa da queda dos gradientes diastólicos pela valva mitral quando comparados os valores após o primeiro e o segundo procedimentos. ${ }^{4}$

O caso relatado torna-se relevante por ser a única descrição de um paciente submetido a VMP por quatro vezes. O aumento da área valvar mitral foi sucessivamente menor a cada VMP realizada nessa paciente (Figura 1), embora o escore de Wilkins tenha sido semeIhante antes de cada intervenção (escores de 9, 10, 10 e 10, respectivamente). Além disso, a intensidade da calcificação valvar e subvalvar também é semelhante nos quatro momentos pré-intervenção.

Outro dado relevante é o tempo necessário para o surgimento de reestenose mitral após a VMP; neste

TABELA 2

Sobrevida livre de eventos após primeira valvoplastia mitral percutânea na população geral

\begin{tabular}{lcc}
\hline & $\begin{array}{c}\text { Tempo de } \\
\text { seguimento (anos) }\end{array}$ & $\begin{array}{c}\text { Sobrevida livre } \\
\text { de eventos (\%) }\end{array}$ \\
\hline Ben Farhat et al. ${ }^{11}$ & 7 & 90 \\
Zaki et al. $^{12}$ & 5 & 91 \\
Saeki et al. $^{13}$ & 6 & 88 \\
\hline
\end{tabular}




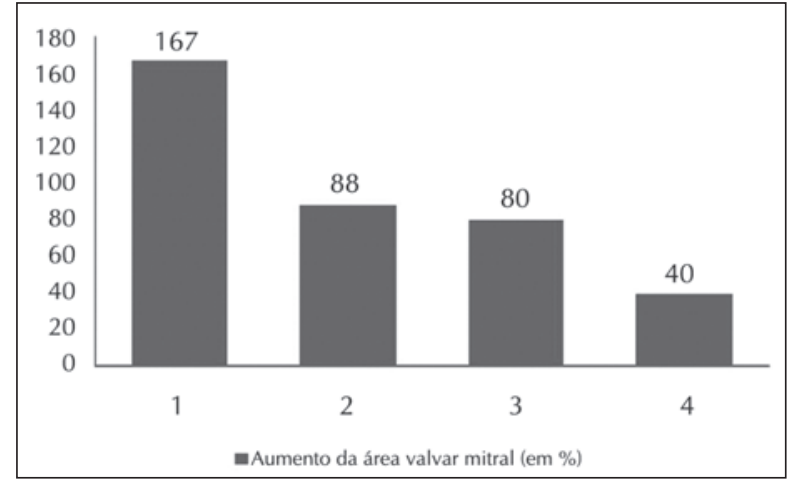

Figura 1 - Aumento porcentual da área valvar mitral pelo ecocardiograma após cada valvoplastia mitral percutânea realizada.

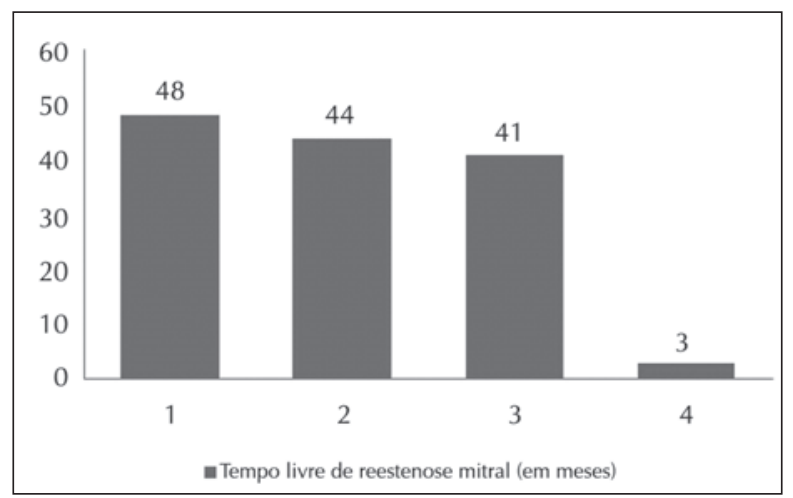

Figura 2 - Tempo livre de reestenose mitral após cada valvoplastia mitral percutânea realizada.

caso, o número de meses livres de reestenose também foi progressivamente menor a cada VMP realizada ( $\mathrm{Fi}-$ gura 2). O sucesso parcial do último procedimento e, consequentemente, a reestenose mitral precoce provavelmente decorreram da maior deterioração das valvas e do aparelho subvalvar, tanto pelo tempo de atividade da doença reumática como por possível fibrose cicatricial provocada pelas dilatações prévias.

Este relato reafirma a possibilidade de sucessivas VMPs em pacientes selecionados que experimentam reestenose mitral, levando-se em consideração a indicação correta de cada procedimento e as chances de sucesso ou complicações. Deve-se ter mente que resultados progressivamente menos satisfatórios devem ser esperados a cada procedimento realizado, pelo grau de complexidade desses pacientes e, possivelmente, pela injúria valvar com consequente fibrose causada por repetidas dilatações com balão.

\section{CONFLITO DE INTERESSES}

Os autores declaram não haver conflito de interesses relacionado a este manuscrito.

\section{REFERÊNCIAS}

1. Inoue K, Owaki T, Nakamura T, Kitamura F, Miyamoto N. Clinical application of transvenous mitral commissurotomy by a new balloon catheter. J Thorac Cardiovasc Surg. 1984; 87(3):394-402.

2. Gomes NL, Esteves C, Braga S, Machado L, Meneghelo Z. Evolução tardia da valvoplastia mitral. Rev Soc Cardiol Estado de São Paulo. 2002;12(2):315-26.

3. Wilkins GT, Weyman AE, Abascal VM, Block PC, Palacios IF. Percutaneous balloon dilatation of the mitral valve: an analysis of echocardiographic variables related to outcome and the mechanism of dilatation. Br Heart J. 1988;60(4):299-308.

4. Gomes NL, Esteves VBC, Braga SLN, Ramos AIO, Esteves FA, Paes AT, et al. Valvotomia mitral percutânea: da primeira à terceira dilatação. Rev Bras Cardiol Invasiva. 2009;17(2):169-75.

5. Abascal VM, Wilkins GT, O'Shea JP, Choong CY, Palacios IF, Thomas JD, et al. Prediction of successful outcome in 130 patients undergoing percutaneous balloon mitral valvotomy. Circulation. 1990;82(2):448-56.

6. Reys VP, Raju BS, Wynne J, Stephenson LW, Raju R, Fromm BS, et al. Percutaneous balloon valvuloplasty compared with open surgical commissurotomy for mitral stenosis. N Engl J Med. 1994;331(15):961-7.

7. Kang DH, Park SW, Song JK, Kim HS, Hong MK, Kim JJ, et al. Long-term clinical and echocardiographic outcome of percutaneous mitral valvuloplasty: randomized comparison of Inoue and double-balloon techniques. J Am Coll Cardiol. 2000;35(1):169-75.

8. Arora R, Kalra GS, Murty GS, Trehan V, Jolly N, Mohan JC, et al. Percutaneous transatrial mitral commissurotomy: immediate and intermediate results. J Am Coll Cardiol. 1994; 23(6):1327-32.

9. Cardoso LF, Ayres CV, Bento AM, Tarasoutchi F, Vieira ML, Grinberg M. Resultados imediatos e tardios da valvoplastia mitral percutânea em pacientes com estenose mitral. Arq Bras Cardiol. 2010;94(3):406-13.

10. Borges IP, Peixoto ECS, Peixoto RTS, Oliveira PS, Netto MS, Labrunie $\mathrm{P}$, et al. Valvoplastia mitral percutânea por balão: evolução a longo prazo e análise dos fatores de risco para óbito e eventos maiores. Arq Bras Cardiol. 2005;84(5):397-404.

11. Ben Farhat M, Ayari M, Maatouk F, Betbout F, Gamra H, Jarrar $M$, et al. Percutaneous balloon versus surgical closed and open mitral commissurotomy: seven-year follow-up results of a randomized trial. Circulation. 1998;97(3):245-50.

12. Zaki A, Salama M, El Masry M, Elhendy A. Five-year follow-up after percutaneous balloon mitral valvuloplasty in children and adolescents. Am J Cardiol. 1999;83(5):735-9.

13. Saeki F, Ishizaka Y, Tamura T. Long-term clinical and echocardiographic outcome in patients with mitral stenosis treated with percutaneous transvenous mitral commissurotomy. Jpn Cir J. 1999;63(8):597-604. 